\title{
Combining EEG and fMRI to investigate the post-movement beta rebound
}

\author{
Laura M. Parkes, ${ }^{*}$ Marcel C.M. Bastiaansen, and David G. Norris \\ F.C. Donders Centre for Cognitive Neuroimaging, University of Nijmegen, Adelbertusplein 1, 6525 EK Nijmegen, The Netherlands
}

Received 28 April 2004; revised 10 August 2005; accepted 19 August 2005

Available online 19 October 2005

\begin{abstract}
The relationship between synchronous neuronal activity as measured with EEG and the blood oxygenation level dependent (BOLD) signal as measured during fMRI is not clear. This work investigates the relationship by combining EEG and fMRI measures of the strong increase in beta frequency power following movement, the so-called post-movement beta rebound (PMBR). The time course of the PMBR, as measured by EEG, was included as a regressor in the fMRI analysis, allowing identification of a region of associated BOLD signal increase in the sensorimotor cortex, with the most significant region in the postcentral sulcus. The increase in the BOLD signal suggests that the number of active neurons and/or their synaptic rate is increased during the PMBR. The duration of the BOLD response curve in the PMBR region is significantly longer than in the activated motor region, and is well fitted by a model including both motor and PMBR regressors. An intersubject correlation between the BOLD signal amplitude associated with the PMBR regressor and the PMBR strength as measured with EEG provides further evidence that this region is a source of the PMBR. There is a strong intra-subject correlation between the BOLD signal amplitude in the sensorimotor cortex during movement and the PMBR strength as measured by EEG, suggesting either that the motor activity itself, or somatosensory inputs associated with the motor activity, influence the PMBR. This work provides further evidence for a BOLD signal change associated with changes in neuronal synchrony, so opening up the possibility of studying other event-related oscillatory changes using fMRI.
\end{abstract}

(C) 2005 Elsevier Inc. All rights reserved.

Keywords: fMRI; BOLD; EEG; Beta rhythms; Event-related synchronization (ERS); Sensorimotor function

\section{Introduction}

Recent work combining EEG and fMRI measurements (Goldman et al., 2002; Laufs et al., 2003a,b; Moosmann et al., 2003) has

\footnotetext{
* Corresponding author. Magnetic Resonance and Image Analysis Research Centre (MARIARC), The University of Liverpool, Liverpool, L69 3GE, UK. Fax: +44 01517945635.

E-mail address: laupar@liverpool.ac.uk (L.M. Parkes).

Available online on ScienceDirect (www.sciencedirect.com).
}

shown that spontaneous changes in alpha and beta power show associated changes in BOLD activity. This suggests that there should also be a BOLD change accompanying event-related changes in oscillatory activity. Any associated BOLD signal change could better localise the source of the oscillatory activity, and the nature of the BOLD change (for example whether the change is positive or negative) could give information on the underlying physiological changes.

The BOLD signal is sensitive to changes in local blood flow, blood volume and oxygen consumption, triggered by changes in neuronal activation. Recent work suggests that the BOLD signal is more sensitive to changes in synaptic input than to spiking output (Logothetis et al., 2001). This is supported by earlier work which, through the careful uncoupling of synaptic input from spiking output, showed cerebral blood flow changes to be independent of spiking output (Mathiesen et al., 1998). This link between the BOLD signal and synaptic input is further supported by evidence that one of the primary pathways for blood flow change is via the vasodilatory action of nitric oxide, whose release is triggered by glutamate release in the excitatory synapses (for review see Attwell and Iadecola, 2002). Event-related changes in the amplitude of oscillatory EEG activity are thought to be caused by changes in synchrony of the underlying neuronal population (Pfurtscheller and Lopes da Silva, 1999). It is not immediately clear how changes in synchrony might cause a change in the average synaptic activity of the neuronal population, and hence a BOLD change, but several mechanisms can be envisaged. If synchrony changes are accompanied by a change in the rate of synaptic events per unit volume, or a change in the amount of vasoactive substances released per synaptic event, then a BOLD signal change would be expected.

One particularly strong event-related oscillatory phenomenon is the post-movement beta rebound (PMBR), where a sharp increase in EEG or MEG power is seen in the beta frequency range (i.e., around $20 \mathrm{~Hz}$ ) following movement offset, with a maximum over the contralateral sensorimotor cortex (Salmelin et al., 1995; Pfurtscheller et al., 1996). Two studies, using MEG and EEG, respectively, have localised the PMBR in primary motor cortex, more specifically to the anterior bank of the central sulcus (Salmelin et al., 1995; van Burik and Pfurtscheller, 1999). Other 
studies using intracerebral recordings in human subjects, observe a broader distribution of PMBR in both the pre- and post-central gyrus (Crone et al., 1998; Ohara et al., 2000; Szurhaj et al., 2003), the frontal medial cortex (Szurhaj et al., 2003) and in the supplementary motor area (Ohara et al., 2000).

The functional significance of the PMBR is unclear. One suggestion is that it represents an 'idling' (Pfurtscheller et al., 1996), or inhibition (Salmelin et al., 1995) of the motor network, possibly by uncoupling the cell assembly that was formed during the planning and execution of the movement. This is supported by studies that locate the PMBR pre-centrally (in motor cortex), and also evidence that the time of the maximum PMBR coincides with a reduced excitability of motor cortex neurons as measured by TMS (Chen et al., 1998). An alternative hypothesis is that the PMBR is involved in sensory reafference, since it occurs after the movement is completed. This is supported by studies showing a more postcentral location of the PMBR (in sensory cortex), and evidence that passive movements (involving no motor cortex activity) produce a PMBR of equal amplitude to active movements, and that this PMBR is abolished on deafferentation (Cassim et al., 2001).

The timing of the PMBR makes it well suited to investigate whether there is a BOLD signal change associated with eventrelated oscillatory activity. The peak in oscillatory power occurs approximately $1 \mathrm{~s}$ after movement offset and has a duration of approximately $2 \mathrm{~s}$ (Neuper and Pfurtscheller, 2001). The PMBR is hence independent in time of movement-related activity and should be detectable as a broadening of the BOLD response.

In the present study, subjects performed repetitive index finger flexions at different rates, for fixed durations, while their EEG and fMRI BOLD signals were measured. The time course of the PMBR, as measured by EEG, is included as a regressor in the fMRI analysis and so can be used to identify any regions with associated BOLD signal change. Given the recent evidence of a BOLD signal change associated with spontaneous changes in beta power (Laufs et al., 2003a,b), we also expect there to be a BOLD signal change associated with an event-related oscillation such as the PMBR. This will provide further evidence that oscillatory changes can lead to changes in blood flow, and so open up a new avenue for combining EEG and fMRI data. The spatial resolution of fMRI is superior to either EEG or MEG, and so our study should provide more precise localisation of the PMBR, giving further insight into its possible function.

\section{Methods}

Subjects

Six healthy, right-handed volunteers (four female, two male, age range $21-33$ ) participated in the study after giving written informed consent. None had any neurological impairment, had experienced any neurological trauma or had used neuroleptics. All subjects were paid a small fee for their participation.

\section{Design and procedure}

The task was to perform right index finger extensions at three different rates ( $1 \mathrm{~Hz}, 2 \mathrm{~Hz}$ and a single finger-extension) cued by a flashing cross on a screen (Fig. 1). The different movement rates potentially improve the power of the study because they make it possible to investigate parametric variations in both the

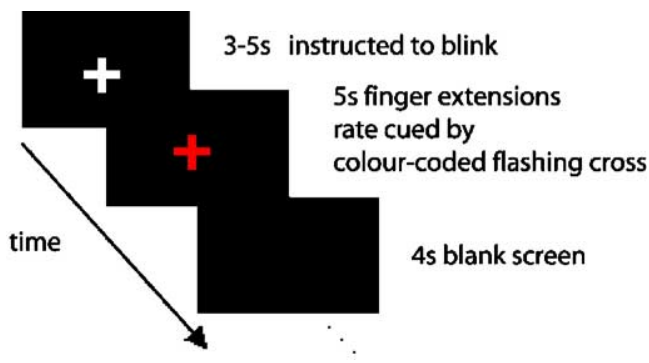

Fig. 1. The visual stimulus. The task was cued by a cross displayed on a screen. Each trial consisted of 3-5 s of a white cross when the subjects were instructed to blink, $5 \mathrm{~s}$ of a flashing coloured cross when finger extensions were performed and $4 \mathrm{~s}$ of a black screen when the beta rebound was expected to occur.

motor and putative PMBR responses. The flashing cross was colour-coded for each movement type to aid in the quick realisation of which extension rate to perform. Subjects were instructed to extend their finger until it made sustained contact with a button fixed above the finger, which was used to record movement onsets and offsets. Movement duration was $5 \mathrm{~s}$ followed by $4 \mathrm{~s}$ of a black screen when the subjects were instructed not to blink, and finally a white cross was displayed for between 3 and $5 \mathrm{~s}$ (randomly jittered between 3, 3.5, 4, 4.5 and $5 \mathrm{~s})$ during which the subjects could blink. Twenty-five trials of each of the three movement rates were randomly interleaved, giving a measurement time of $15 \mathrm{~min}$. This was repeated three times, giving a total measurement time of approximately $50 \mathrm{~min}$. The EEG measurements were performed both outside and inside the MRI scanner (during continuous, simultaneous scanning) using the same stimulus equipment. Four subjects performed the measurement first inside the scanner and then outside, with the order reversed for the other two subjects.

\section{EEG recordings}

EEG was acquired using the MRI-compatible BrainAmp MR (Brainproducts, Munich, Germany) EEG amplifier and the BrainCap electrode cap (Falk Minow Services, Herrsching-Breitbrunn, Germany) with sintered $\mathrm{Ag} / \mathrm{AgCl}$ ring electrodes providing 29 EEG channels, 2 ECG channels and 1 EOG channel. They were positioned according to the $10 / 20$ system. The reference electrode was between $\mathrm{Fz}$ and $\mathrm{Cz}$. A $250-\mathrm{Hz}$ hardware filter was placed between the electrode cap and the EEG amplifier. The EEG was recorded with a $0.3 \mathrm{~s}$ time constant and a $70 \mathrm{~Hz}$ low-pass filter (for recordings outside the scanner only), and was continuously sampled at $500 \mathrm{~Hz}$ (outside the MR scanner) or at $5 \mathrm{kHz}$ (inside the MR scanner). All recordings were done with the Brain Vision Recorder software (Brainproducts).

\section{Image acquisition}

MRI measurements were performed using a $3 \mathrm{~T}$ Trio wholebody scanner (Siemens, Erlangen, Germany). A birdcage resonator was used for RF transmission and signal reception. The maximal gradient strength was $40 \mathrm{mT} / \mathrm{m}$. Images were acquired using a standard gradient-echo EPI sequence (TR $2 \mathrm{~s}$ including $56 \mathrm{~ms}$ dead time, TE $36 \mathrm{~ms}, 27$ slices, isotropic voxels of $3.5 \mathrm{~mm}$ resolution) covering the whole brain. A 3D MPRAGE (Mugler and Brookeman, 1990) sequence with $2 \mathrm{~mm}$ isotropic resolution was used for the anatomical scan. 


\section{EEG data analysis}

Artefact correction for EEG data recorded inside the scanner

For the EEG data recorded within the MRI environment, the scan and pulse artefacts were corrected along the lines described by Allen et al. (1998, 2000), using Brain Vision Analyzer software (Brainproducts). For the correction of scan artefacts, a pulse from the MR scanner marked the onsets of contaminated EEG segments. The intervals from volume scan onset to volume scan offset were averaged, and their means were subtracted from each individual interval. In addition to the adaptive filtering, inherent to the subtraction method, a $35-\mathrm{Hz}$ low-pass filter was applied to the data, after which the EEG was down-sampled to $500 \mathrm{~Hz}$. For the correction of pulse artefacts, a similar subtraction procedure was used, following identification of the R peaks in the ECG data.

\section{Analysis of the beta rebound}

This analysis was applied to the EEG data recorded both outside the scanner and inside the scanner following artefact correction as detailed above. In order to quantify the PMBR, the EEG data collected during the performance of the movement task were segmented with respect to movement offset (defined as the time at which the response button was released). EEG segments ranging from $1 \mathrm{~s}$ before to $4 \mathrm{~s}$ after movement offset were baselinecorrected (from $1 \mathrm{~s}$ before movement offset to movement offset), and segments containing residual scanner, pulse, eye movement or other artefacts were excluded from further analysis.

For a time-frequency analysis of the EEG data, we used a multi-taper approach (Mitra and Pesaran, 1999). Power was analysed between 1 and $60 \mathrm{~Hz}$, in $1-\mathrm{Hz}$ steps, as a function of time and frequency using a sliding window technique. Window
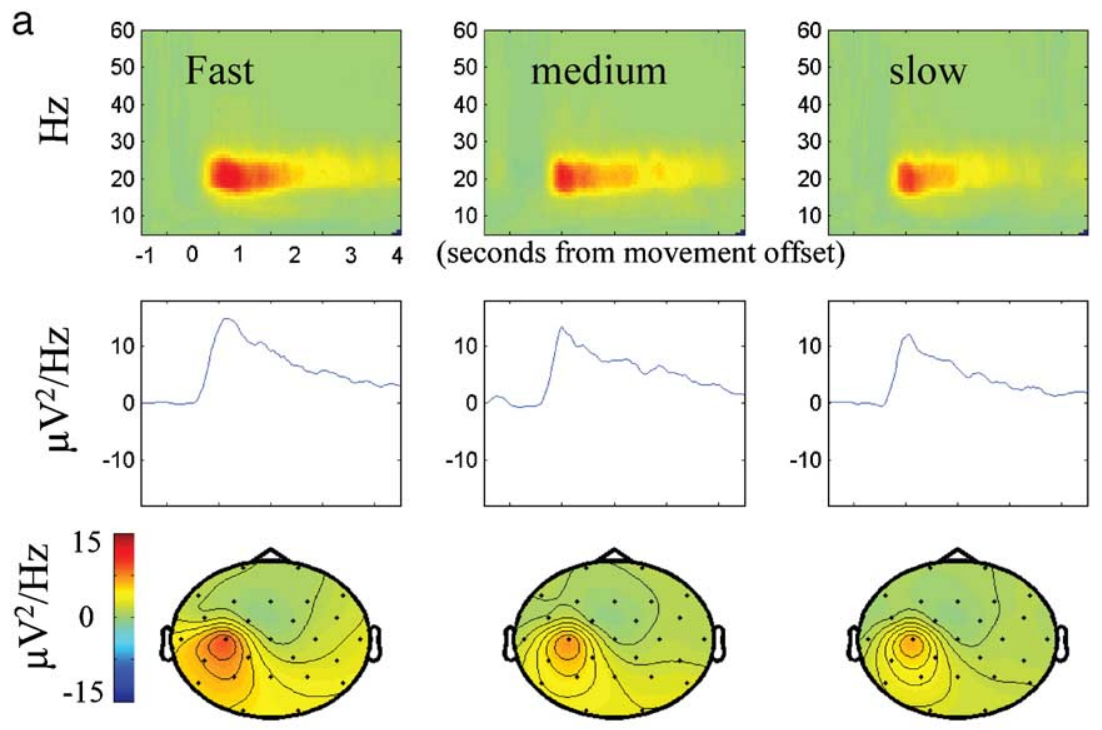

$\mathrm{b}$
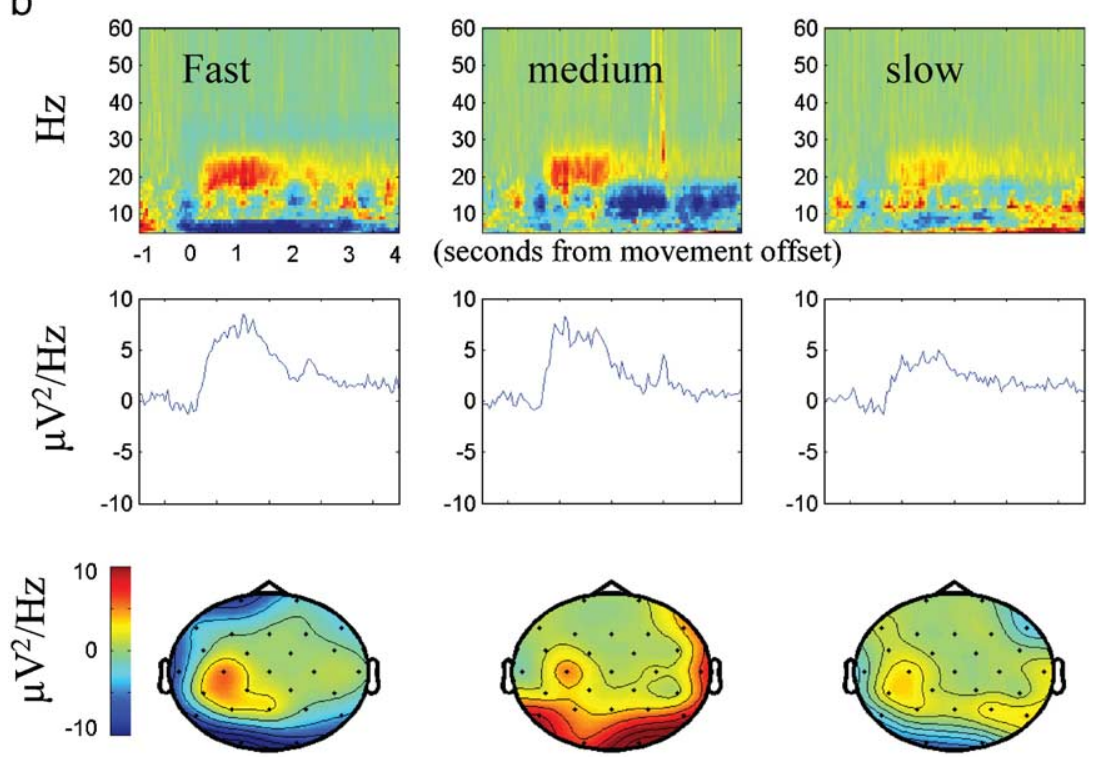

Fig. 2. Time-frequency analysis of the EEG data for one subject (subject 1) both outside (a) and inside (b) the scanner. Top row: time-frequency representation from electrode C3. The PMBR can be clearly seen in all 3 conditions. The second row shows the extracted waveforms (profiles) over the selected frequency range, which are used to create the PMBR regressor. The third row shows the topography of the PMBR for each condition. For all six subjects, the source of the PMBR is located in the contralateral sensorimotor cortex. 
lengths were adapted to the different frequencies so as to contain 7 cycles. This yields a time-frequency resolution trade-off similar to that obtained with Morlet wavelets. Multi-tapers were chosen to concentrate spectral energy over frequency bands that widened with increasing frequency, such that for a given frequency, concentration was over a band of $\pm 1 / 5$ of that frequency.

Power changes were then expressed as the absolute change in power (in $\mu \mathrm{V}^{2} / \mathrm{Hz}$ ) relative to the 1 -s pre-offset baseline. It was not possible to use a pre-movement baseline, as is standard practice, since these data were contaminated by artefacts due to blinking. This will result in an increase in the amplitude of the PMBR compared to previously published results, due to beta desynchronisation during movement. For each subject, we determined, through visual inspection of the time-frequency representations (see Fig. 2a, top row) at electrode C3 (i.e., overlying the contralateral sensorimotor cortex), the frequency band that best characterised the PMBR. Power changes at electrode C3 were averaged within these subject-specific frequency bands, and the resulting profiles (see Fig. 2a, middle row) were used as regressors in the fMRI analysis. The integral of the PMBR profile was calculated for use as a measure of the 'strength' of the PMBR EEG signal. We refer to this as 'PMBR strength'.

\section{fMRI data analysis}

Standard MRI pre-processing was carried out using BrainVoyager (Brain Innovation, Maastricht) including motion correction, slice time correction, spatial and temporal smoothing and transformation into Talairach space. Four regressors were used in a general linear model: three motor regressors corresponding to the three extension rates (onsets and offsets of the movement block determined from the button presses), and a PMBR regressor constructed from the averaged PMBR profiles (Fig. 3). For group analysis, the PMBR regressor was scaled according to the strength of each subject's PMBR. The relative amplitudes of the independent regressors (i.e., PMBR regressor compared to the motor regressors) are unimportant since they will only influence the beta weight of each regressor $\left(\beta_{1}, \beta_{2}\right.$, etc. in Fig. 3$)$ but not the statistical significance of the BOLD signal associated with each

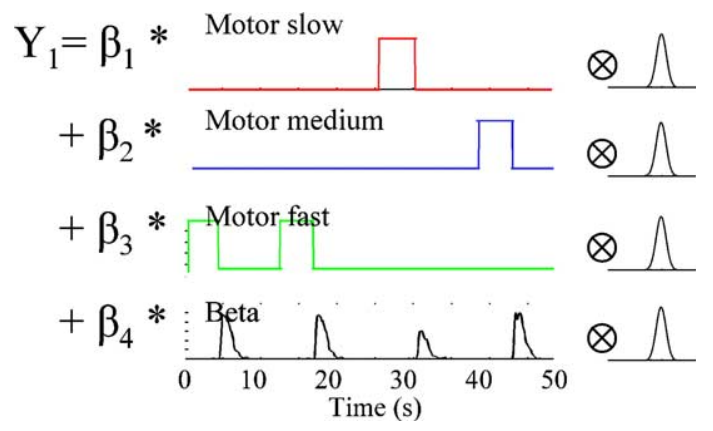

Fig. 3. Regressors for the general linear model, shown for $50 \mathrm{~s}$ of typical data. There are three motor regressors and one PMBR regressor that is composed of the average PMBR profiles as measured outside the scanner (see Fig. 4a middle row), with the relative amplitudes of each profile preserved. The motor regressors represent blocks of movement rather than individual finger extensions, with onset and offset determined by the first press and final release of the response button for each movement block. For group analysis, the amplitude of the PMBR regressor is scaled for each subject according to the maximum power of his or her rebound. Each regressor is convolved with a Gaussian HRF.

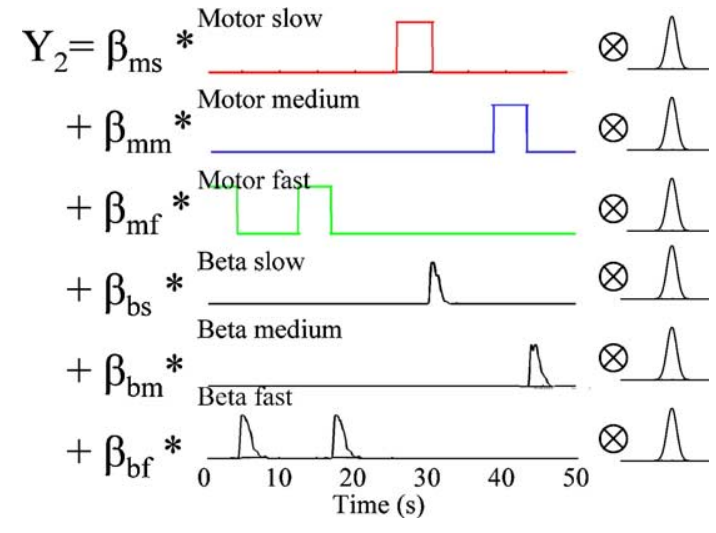

Fig. 4. Regressors for the general linear model used within regions of interest. There are 3 motor regressors and 3 PMBR regressors. All regressors are scaled to uniform amplitude. The fitted beta weights ( $\beta_{\mathrm{bs}}$, etc.) will then reflect the amplitude of the MRI signal that is associated with each regressor. Each regressor is convolved with a Gaussian HRF.

regressor. The regressors were convolved with a Gaussian haemodynamic response function (HRF) with a delay of $4.5 \mathrm{~s}$ and dispersion of $4.7 \mathrm{~s}^{2}$ (equivalent to a full width half maximum (FWHM) of 5 s) (Rajapakse et al., 1998).

The model was used in both individual and group analyses to identify regions where all three movements account for significant variance in the MRI data ('motor regions'), and also where the PMBR regressor accounts for significant variance in the MRI data ('PMBR regions').

In any PMBR regions identified, a second general linear model is used to establish the amplitude of the MRI response associated with each particular PMBR condition. In this second model, there were six regressors: three motor regressors as before and three PMBR regressors, corresponding to the rebounds following each extension rate (Fig. 4). All PMBR regressors were normalised to the same maximum amplitude so that the beta weights ( $\beta b s, \beta b m, \beta b f$ in Fig. 4) fitted during the analysis will indicate the amplitude of the MRI signal associated with each particular PMBR regressor. We refer to the BOLD response associated with the PMBR regressor as the 'PMBR BOLD activity' (and 'PMBR BOLD amplitude' refers to the associated beta weight) and likewise 'motor BOLD activity' for signal associated with the motor regressor.

The average BOLD signal time course in each region of interest was also found. This was calculated in terms of the percentage BOLD increase with respect to a baseline set between 5 and $3 \mathrm{~s}$ before movement offset.

\section{Results}

\section{EEG results}

All six subjects showed a PMBR for all three movement conditions, with peak frequency varying between 15 and $25 \mathrm{~Hz}$ (mean $20 \mathrm{~Hz}$ ). The EEG data recorded inside the scanner were not of sufficient quality for use in the analysis, with approximately five times less signal to noise than that recorded outside the scanner. However, for two out of six subjects (subjects 1 and 4), the rebound could be identified inside the scanner and it was found to be qualitatively the same as that recorded outside the scanner (Fig. 2). This suggests that the PMBR was stable between the two 


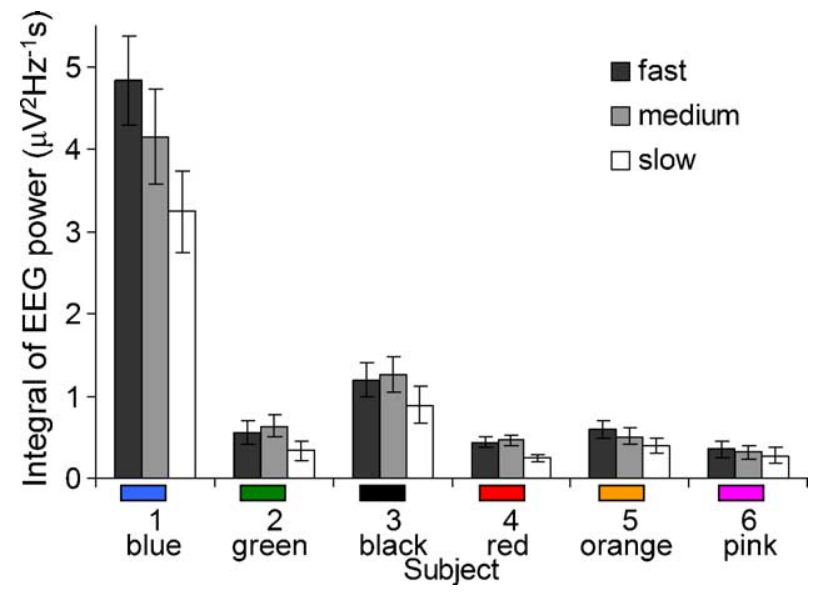

Fig. 5. Parameterisation of the PMBR with stimulus condition. The integral of the PMBR profile for the three movement conditions (fast, medium and slow) is shown for all six subjects. The three conditions changed the strength of the PMBR differently across subjects. However, the slow movement condition always produced the lowest PMBR power. Subject 1 has an unusually large EEG response. The error bars show the standard error in the mean, calculated using a jack-knife procedure on the single trials of each condition. Each subject is identified with a colour (given under the chart), which is consistently used in subsequent figures.

measurements for all subjects. Support for this comes from studies showing high reproducibility of the PMBR in individual subjects over time (see e.g., Pfurtscheller and Lopes da Silva, 1999). On this basis, we chose to use the higher quality data from outside the scanner in our analysis.

Fig. 5 shows that while different movement conditions did manipulate the strength of the PMBR, the variation within each subject was small. However, the average power of the rebound varied strongly between subjects, allowing intersubject comparisons of the PMBR. Similar topography (row three of Fig. 2) of the rebound was observed across all subjects and conditions.

\section{Motor BOLD activity}

Fig. 6 shows the results of the group analysis, highlighting regions where all three motor regressors account for significant variance in the fMRI data. By increasing the threshold of activation (Fig. 6b), it can be seen that the most significant region is located on the anterior bank of the 'omega-shaped' part of the central sulcus, with Talairach coordinates $(-33-2851)$. Previous studies confirm this to be the location of the motor hand area (Yousry et al., 1997).

The duration of the motor BOLD response (averaged over the three conditions) was consistent across subjects and regions. The BOLD response from the focus of motor activation in each individual subject has a mean FWHM of $6.5 \mathrm{~s}$ and standard deviation of $0.3 \mathrm{~s}$ across subjects. Within the activated motor region (excluding the PMBR region), the duration of the BOLD response was measured in six regions of $125 \mathrm{~mm}^{3}$ for each subject. The duration was found to be very stable, with a mean standard deviation of the FWHM of $0.2 \mathrm{~s}$ (standard deviation $0.07 \mathrm{~s}$ across subjects).

Fig. 7 shows the correlation of the motor BOLD amplitude within the focus of the motor region (yellow region on Fig. 6b) with the PMBR strength as measured by EEG. Fig. 7a shows that there is no significant correlation across subjects, i.e., subjects with generally high PMBR strength do not show a generally high BOLD response in the motor region (the weak correlation seen in Fig. 7a disappears on removal of the outlying subject, subject 1).
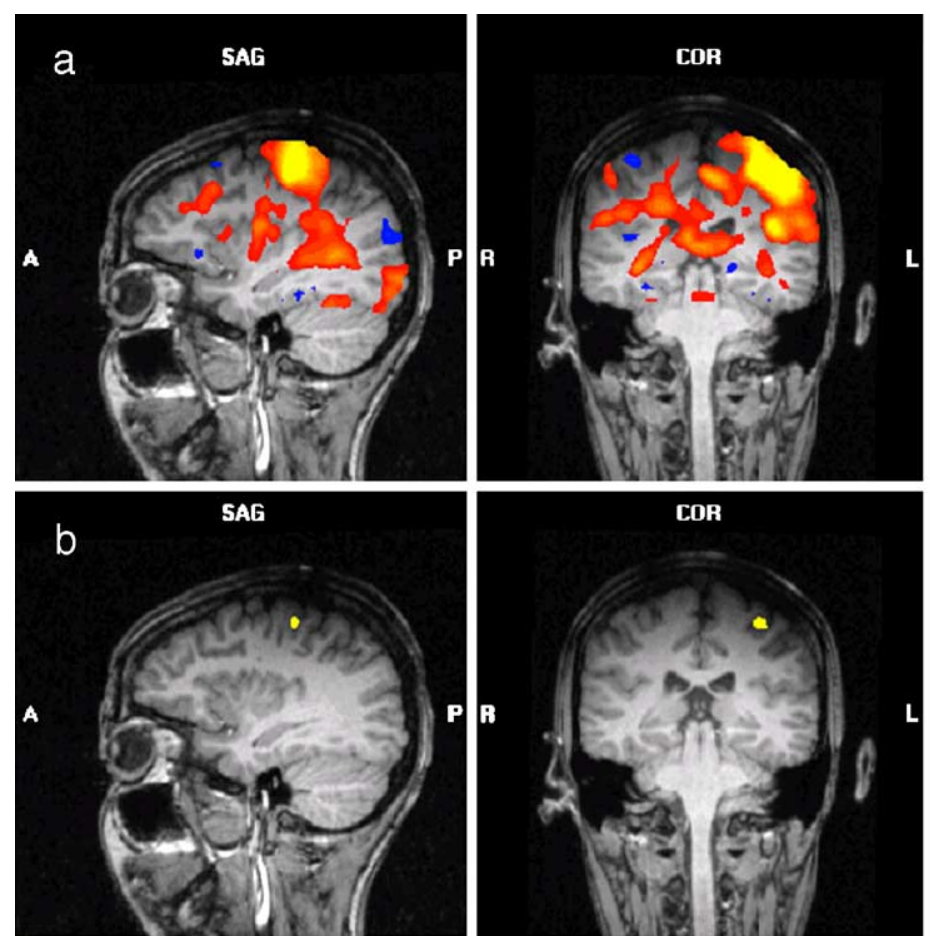

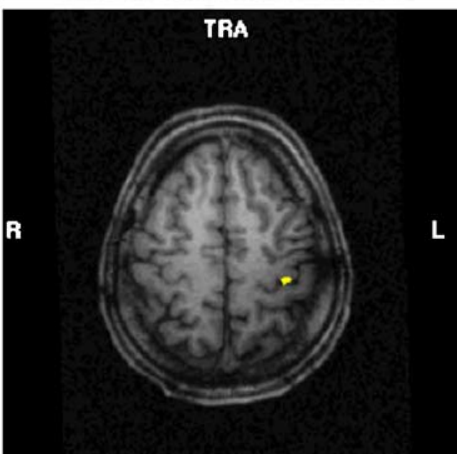

Fig. 6. Regions of motor activation. Motor activation regions over all six subjects, overlaid on the anatomical image of one subject in Talairach space. The coloured voxels highlight regions where all three motor regressors accounted for significant variance in the MRI signal. Panel a shows significant regions with $P<0.05$ (Bonferroni corrected) and panel b shows the most significant region, which is located in the central sulcus with Talairach coordinates (-33 -2851$)$. 
a

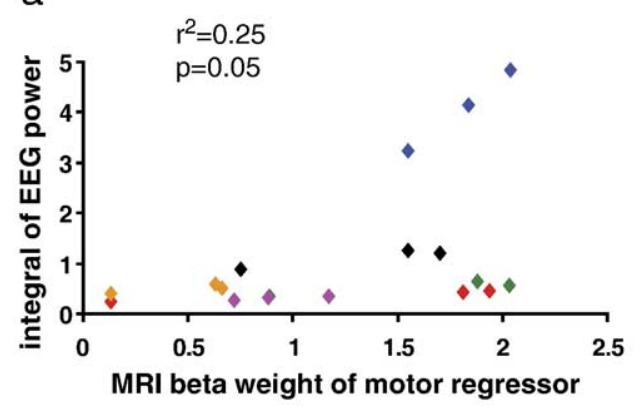

b

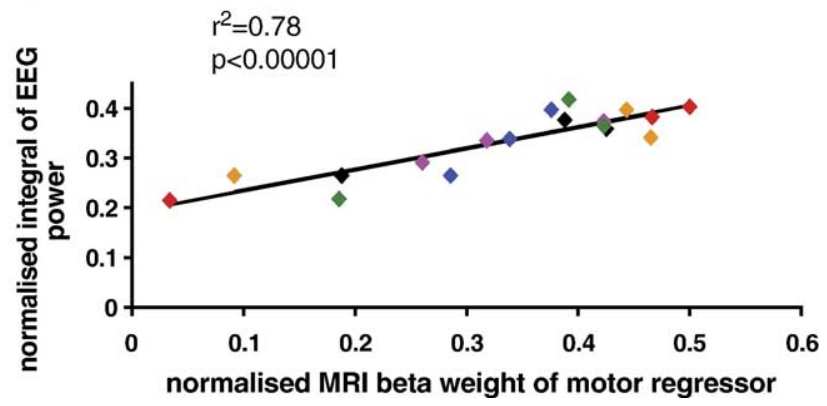

Fig. 7. Correlations between motor BOLD activity and PMBR strength in the motor region. The amplitude of the MRI response in the motor region (given by the beta weight of the motor regressor) for all subjects (each shown with a different colour) and all conditions is correlated against the PMBR strength, as measured with EEG. The weak correlation seen in the original data (a) disappears on removal of the outlying subject. However, on normalisation of both the MRI and the EEG response, a very strong correlation is seen within individual subjects (coefficient of determination $r^{2}=0.78, P<0.0001$ ). The significance of correlation was determined using a two-tailed $t$ distribution.

However, by normalising both measurements to the average response for each subject, it is possible to consider the correlation over the three conditions within each subject. This shows a strong correlation (Fig. 7b) between motor BOLD activity and the PMBR.

\section{PMBR BOLD activity}

Fig. 8 shows the results of the group analysis, highlighting regions where the PMBR regressor accounts for a significant degree of variance in the fMRI data. A region of positive correlation can be seen in the left sensorimotor cortex, which we identify as the source of the PMBR. The region of significant activation $(P<0.05$, Bonferroni corrected) encompasses both motor cortex and somatosensory cortex (Fig. 8a). Increasing the threshold of significance (Fig. 8b) shows the most significant region of PMBR BOLD activity (red) in relation to the focus of motor BOLD activity (yellow). It can be seen that the focus of the PMBR activation is located in the post-central sulcus, with Talairach coordinates $(-51-3149)$.

Individual analysis revealed a region with significant positive correlation with the PMBR regressor in three out of the six subjects ( $P<0.05$, Bonferroni corrected). These three subjects (subjects 1,2 and 3) were those with the largest PMBR as measured with EEG. The location of this region in each of these three subjects is consistently found in the post-central sulcus as shown in Fig. 9. The Bonferroni correction is quite conservative, so we considered the effect of lowering the threshold of activation on the remaining three subjects. All three subjects showed a discrete region of positive
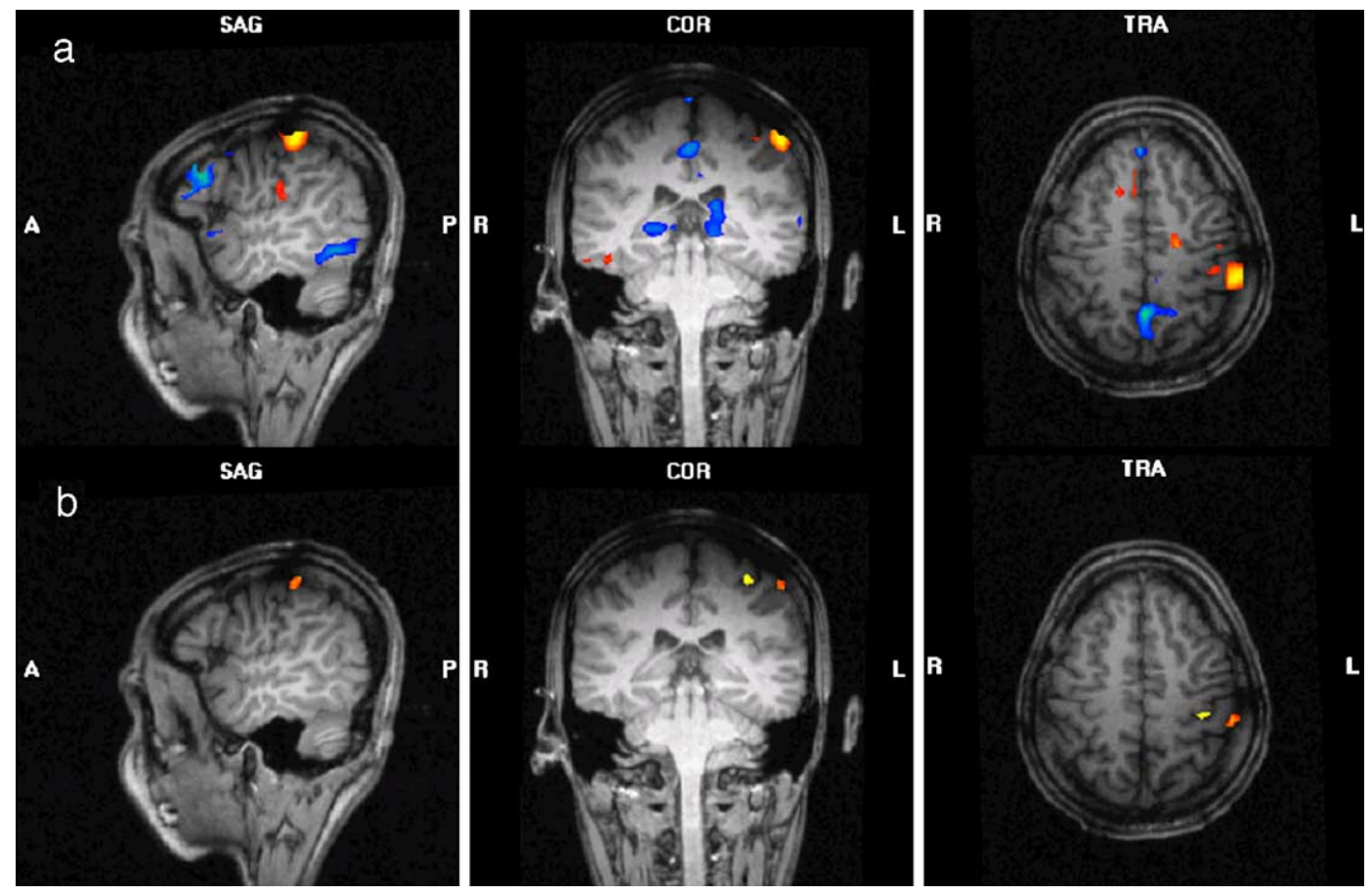

Fig. 8. Regions of PMBR activation. PMBR activation regions over all six subjects, overlaid on the anatomical image of one subject in Talairach space. The coloured voxels highlight regions where the PMBR regressor accounted for significant variance in the MRI signal. Panel a shows significant regions with $P<0.05$ (Bonferroni corrected). Panel b shows the most significant PMBR region (red) alongside the most significant motor region (yellow) from Fig. 6b. It can be seen that the focus of motor activity is in the central sulcus and the focus of the PMBR is in the post-central sulcus, with Talairach coordinates $(-51-3149)$. 

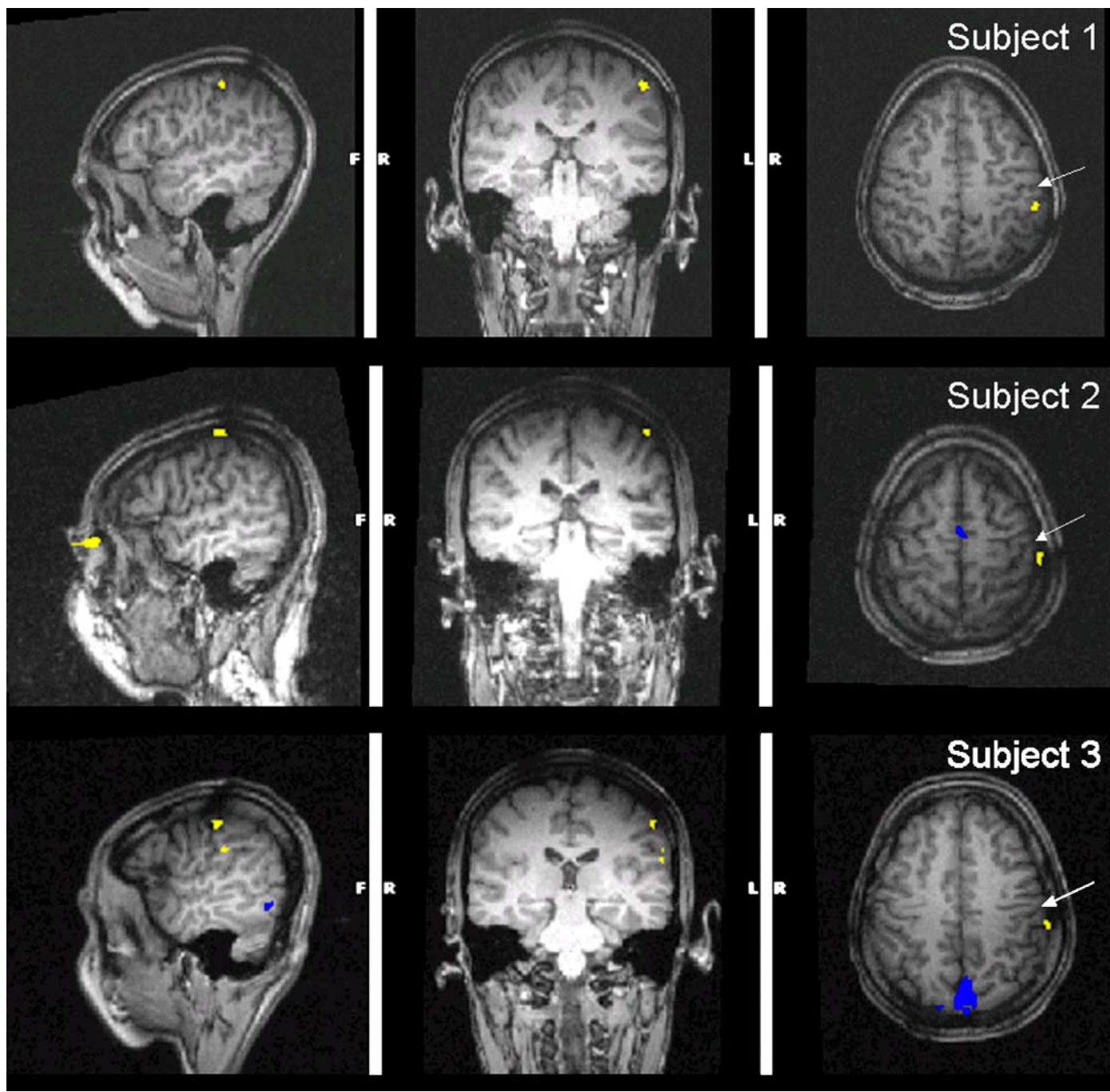

Fig. 9. Individual regions of PMBR activation. PMBR activation regions from three individual subjects (subjects 1,2 and 3), overlaid on the anatomical image from each individual subject. The coloured voxels highlight regions where the PMBR regressor accounted for significant variance in the MRI signal. In each case, the focus of the PMBR activity is in the lateral part of the post-central sulcus. The central sulcus is marked with an arrow.

correlation in the sensorimotor cortex $(P<0.00001$, uncorrected), with two located post-centrally and one in the central sulcus.

Figs. 8 and 9 also show several regions with a significant signal decrease during the PMBR, in the midline and widespread in the occipital cortex. The signal in the occipital cortex can be explained by the time course of the visual stimulus (see Fig. 1). During the $4 \mathrm{~s}$ post-movement over which the PMBR occurs, the screen was blank, whereas at all other times, there was a cross present. The absence of visual stimulation over this period can account for the negative correlation seen in the occipital cortex. The midline regions showed no significant correlation with the PMBR strength, suggesting that these regions are not involved in the PMBR.

To check that the results are not dominated by the single subject with the largest EEG response (subject 1), the analysis was repeated using only the remaining five subjects. Fig. 10 shows significant PMBR activation ( $P<0.05$, Bonferroni corrected) for the five-subject analysis in light blue. The five-subject analysis also located PMBR activity in the post-central sulcus, but more anterior and inferior to the six-subject analysis (red).

Fig. 11 shows the average signal time course from the regions of PMBR activation in the three subjects shown in Fig. 9 (i.e., the average signal from each subject in their individually activated region), averaged over all conditions. This can be compared to the time course for motor activation taken from the individual motor regions of these three subjects. It can be seen that the signal in the PMBR region extends for approximately $2 \mathrm{~s}$ longer (the FWHM is approximately $1 \mathrm{~s}$ longer) than the signal in the focus of motor activation, suggesting a contribution from both the movement and the PMBR in this region. The divergence of the BOLD signals from the two regions begins approximately $1 \mathrm{~s}$ prior to movement offset. We believe that this reflects the different motor response in the two regions, but does not exclude a later PMBR component in the PMBR region. Fig. 11 also shows the average model signal curves from these three subjects, using the appropriate regressors convolved with a Gaussian HRF. Fig. 11a shows modelling using standard parameters (Gaussian HRF with delay time $4.5 \mathrm{~s}$ and dispersion $4.7 \mathrm{~s}^{2}$ ), whereas Fig. 11b uses a shorter delay time $(4.0 \mathrm{~s})$, which appears to provide a better fit. The signal from the motor region is well-modelled by motor regressors alone (orange line), whereas a model with both motor and PMBR regressors fits the data in the PMBR region well (blue line). Re-analysis using the delay time of $4.0 \mathrm{~s}$ identified very similar activated regions (but with higher 

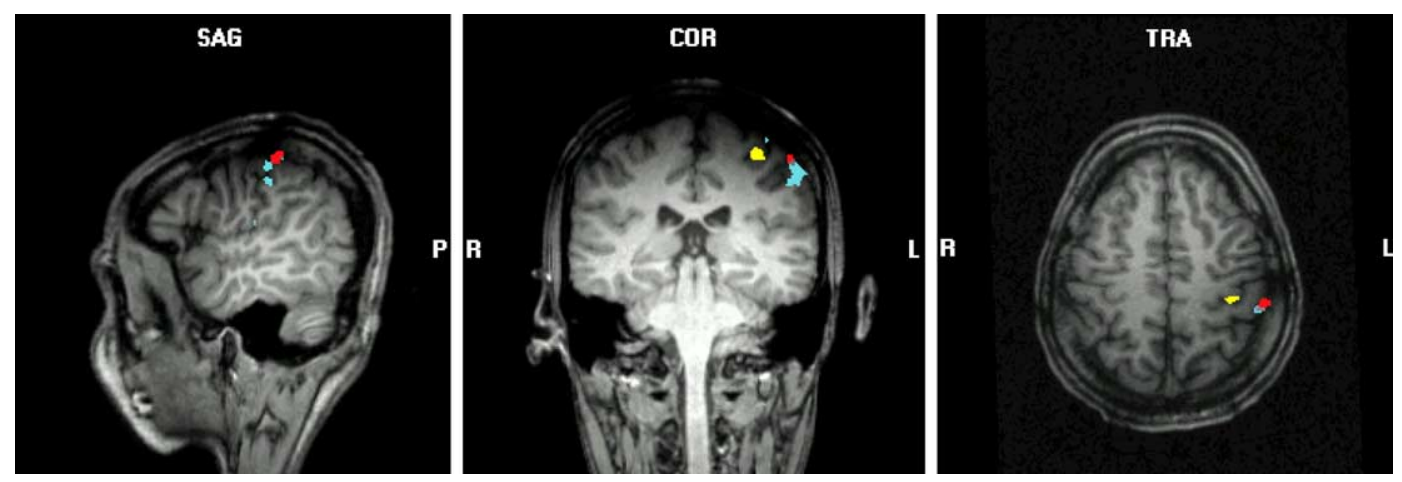

Fig. 10. Five-subject region of PMBR activation. The region of PMBR activation for a five-subject group analysis (excluding subject 1 with the largest PMBR as measured with EEG) is shown in light blue. The results are shown at significance level $P<0.05$ (Bonferroni corrected), and are displayed alongside the most significant six-subject PMBR region (red) from Fig. $8 \mathrm{~b}$ and the most significant motor region (yellow) from Fig. 6b. The focus of the five-subject PMBR region has Talairach coordinates $(-56-2643)$.

significance as to be expected) but showed no meaningful change in the results of the subsequent correlation analysis, and hence the standard parameters were employed throughout.

\section{Correlations between BOLD activity and PMBR strength within the PMBR region}

Fig. 12 shows the correlations of the BOLD amplitude within the PMBR region (red region on Fig. $8 \mathrm{~b}$ ) with the PMBR strength. In this region, both the motor regressors and the PMBR regressors account for significant variance in the data, so both the early motor component of the BOLD signal and the later PMBR component are considered. For this analysis, the second general linear model (Fig. 4 ) is used to find the beta weights of all six regressors in the PMBR region. As was found in the motor region, the motor BOLD amplitude shows no correlation with PMBR strength across subjects (Fig. 12a), but a strong correlation within subjects (Fig. $12 \mathrm{~b}$ ), found by normalising the data. However, the PMBR BOLD amplitude, reflecting the later component of the BOLD signal, shows opposite behaviour with no significant correlation within subjects (Fig. 12d), but a significant correlation across subjects (Fig. 12c, this correlation has the same significance on removal of the outlier, subject 1). To correct for the fact that the data points are not independent, we averaged the data over all conditions for each subject; the correlation remained significant $(P<0.05)$. This shows that subjects with a generally high PMBR strength also have a generally high PMBR BOLD amplitude.

\section{Discussion}

We have found evidence for a BOLD signal change associated with the PMBR. The signal is located post-centrally, in agreement with the hypothesis that the PMBR is more related to sensory afference than to inhibition of the motor cortex. We have also shown that BOLD signal changes during movement correlate with the PMBR, suggesting an influence of motor cortex activity, or somatosensory input on the subsequent PMBR. We will now discuss this evidence in more detail.

\section{Evidence for a BOLD signal increase associated with the PMBR}

Figs. 8 and 9 show evidence for the existence of a BOLD signal change associated with the PMBR. The region of significant activation $(P<0.05$, Bonferroni corrected) extends over both the motor cortex and somatosensory cortex. For group results over all
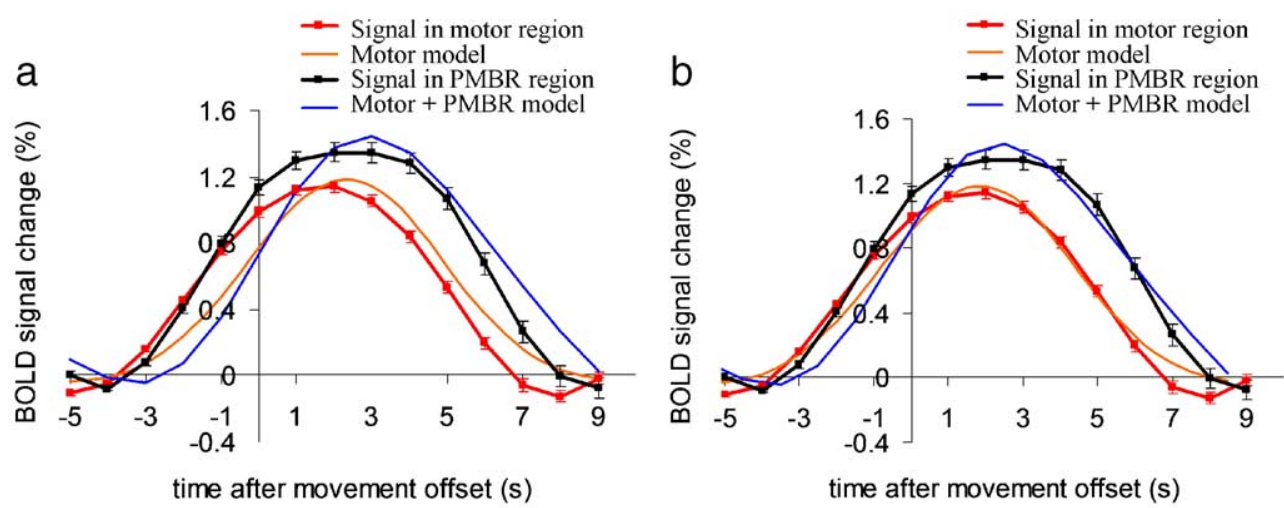

Fig. 11. The BOLD signal in the activated regions. The BOLD signal curve averaged across all conditions and three subjects (subjects 1,2 and 3 ) for the motor region (red) and the PMBR region (black; regions as shown in Fig. 9). The percentage BOLD increase is calculated with respect to a baseline set between 5 and $3 \mathrm{~s}$ before movement offset. The error bars show the standard error across all measurements. The orange line shows the model signal using only motor regressors convolved with a Gaussian HRF, and the blue line is the model signal when using both motor and beta regressors. Panel a shows the results using a Gaussian HRF with a delay time of $4.5 \mathrm{~s}$ and dispersion of $4.7 \mathrm{~s}^{2}$ (as used in the analysis) and panel b shows the results with a shorter delay time of $4.0 \mathrm{~s}$ and the same dispersion. This shorter delay time provides a better fit to the data. 
Motor regressor
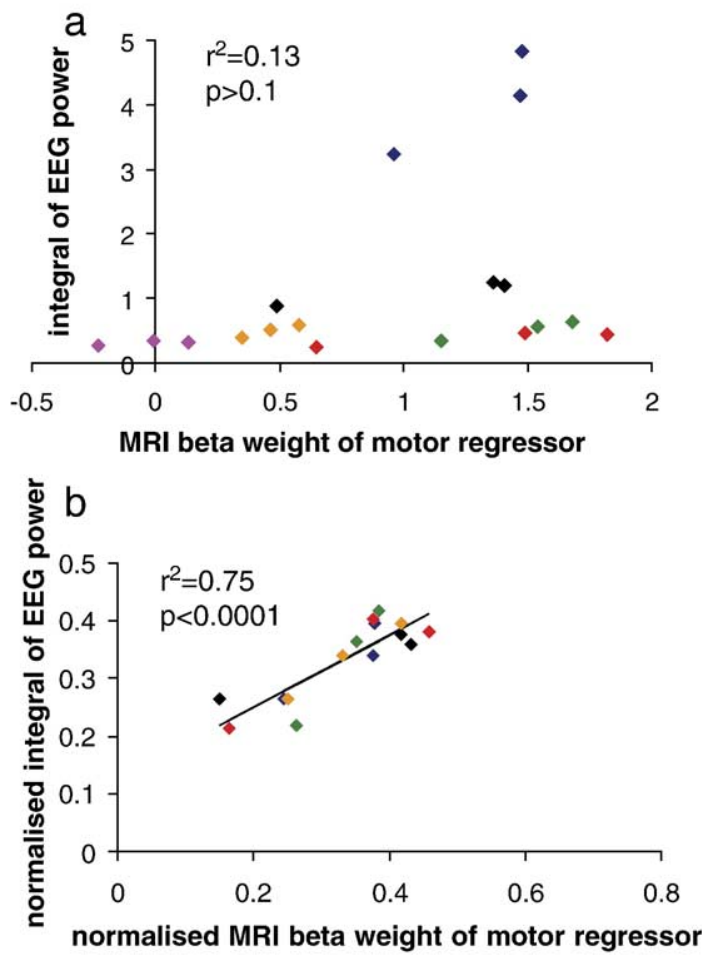

PMBR regressor
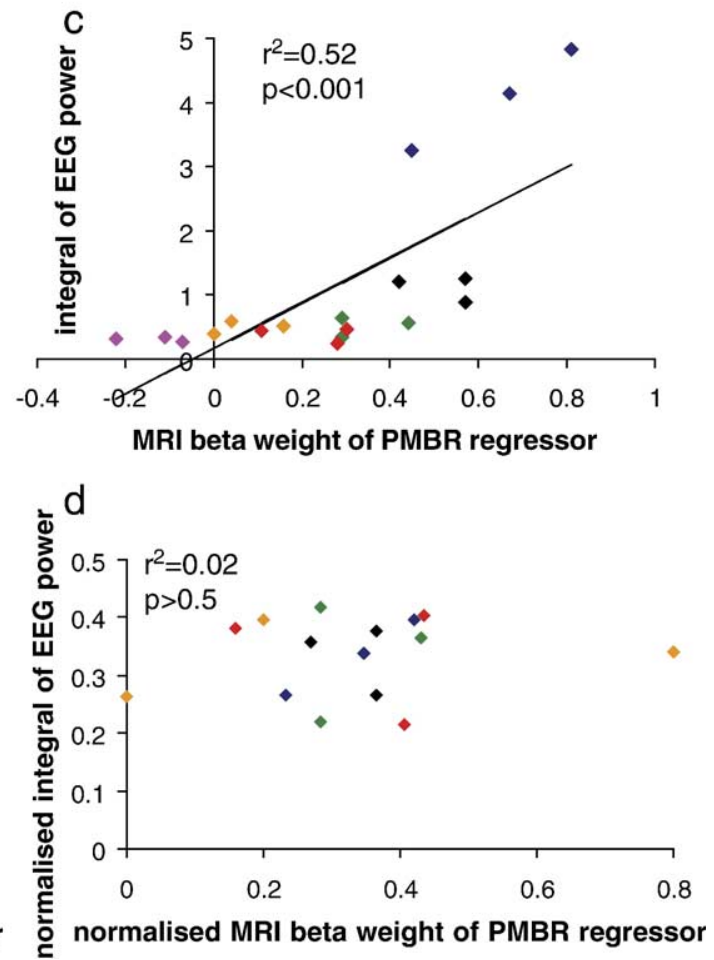

Fig. 12. Correlations between BOLD activity and PMBR strength in the PMBR region. Correlations between the EEG power and the beta weights of the motor regressor $(\mathrm{a}, \mathrm{b})$ and the PMBR regressor $(\mathrm{c}, \mathrm{d})$ in the PMBR region of the group analysis (red, Fig. 8b) are shown across all subjects and conditions. There is no significant correlation across subjects for the motor response (a), but there is a strong correlation within each subject across the three conditions, as shown in the normalised data (b). The BOLD activity associated with the PMBR regressor shows the opposite behaviour, with a significant correlation with the EEG power across subjects (c), but no correlation within each subject across conditions (d). Note that the normalised results show only 5 subjects due to the difficulty in normalising the data from the subject with a negative MRI beta weight (subject 6). The significance of correlation was determined using a twotailed $t$ distribution.

six subjects and individual results in five subjects, the most significant region of PMBR activation is found in the contralateral post-central sulcus. Re-analysis on removal of the subject with the strongest PMBR (subject 1) showed a similar pattern of BOLD activation (Fig. 10), indicating that the group result is not dominated by this subject.

The PMBR BOLD amplitude is significantly correlated with PMBR strength across subjects (Fig. 12c), supporting the identification of this region as the focus of the rebound. This positive correlation, and the relative timing of this BOLD component, suggests that the PMBR BOLD signal and the PMBR as measured by EEG are measurements of the same underlying neuronal activity. While each subject shows quite a different baseline beta power (due to different sources of noise at the beta frequency during the experiment), the PMBR strength is a measure of power change, which should reflect the increase in number and/ or degree of synchronisation of the underlying neurons. If the neurovascular coupling between the underlying PMBR activity and the PMBR BOLD is similar in each subject, this should then be reflected as a correlation between PMBR BOLD amplitude and PMBR strength both within and across subjects. While there was a significant intersubject correlation between the PMBR BOLD amplitude and PMBR strength, this relationship was not maintained within individual subjects. The intersubject differences in PMBR strength are larger than the intra-subject differences, so it appears that the parameterisation of the PMBR was not large enough to test the relationship within individual subjects given the noise in the BOLD data.

Since the region of PMBR is located within a broad region of motor activation (Fig. 6a), it is important that the contribution from the motor and PMBR regressors can be distinguished. Central to this is the dispersion parameter of the HRF, which we chose carefully in consideration of previous research $\left(4.7 \mathrm{~s}^{2}\right.$, equivalent to a FWHM of $5 \mathrm{~s}$; Rajapakse et al., 1998). This value is the same as that suggested by Glover (1999), and is similar to the $4.7 \mathrm{~s}$ FWHM suggested by Soltysik et al. (2004). It can be seen (Fig. 11) that the chosen dispersion value does appear to correctly model our data. The BOLD response in the PMBR region has a FWHM of approximately $1 \mathrm{~s}$ longer than in the motor region (Fig. 10), as predicted by the model including both a motor and a PMBR regressor (Fig. 10, blue line).

It is possible that the broadening of the BOLD signal in this region merely reflects a broader $\mathrm{HRF}$ and is not related to the PMBR. While there is some variability in the width of the HRF between regions, we would not expect it to be as large as $1 \mathrm{~s}$. Indeed, across the activated motor region, the FWHM had a standard deviation of only $0.2 \mathrm{~s}$. Furthermore, this explanation cannot account for the correlation between PMBR strength and PMBR BOLD activity across subjects. It is important to note that, unlike the PMBR BOLD component (Fig. 12c), the motor BOLD amplitude in this region does not show intersubject correlation (Fig. 12a), strengthening the argument that there are two 
independent, but overlapping components of BOLD activity in this region, rather than a single motor response with a more dispersed HRF than in the primary motor cortex.

\section{Involvement of the sensorimotor cortex in generation of the PMBR}

Fig. 7b shows a strong intra-subject correlation between the BOLD motor amplitude in the motor cortex and the PMBR strength. That is, within each subject, the motor cortex activity strongly predicts the strength of the subsequent PMBR. This suggests that the motor cortex is involved in the generation of the PMBR, a notion supported by previous work showing a correlation between PMBR strength and motor cortex activity through varying the external load during a movement (Stancak et al., 1997), or increasing the size of the muscle activated (Pfurtscheller et al., 1998). There are of course other factors that could be correlated with the motor activity that could instead be influencing the PMBR. For example, the faster movement conditions associated with a greater motor activity are also likely to have increased somatosensory input. This is supported by the fact that the movement-related signal in the sensory cortex (Fig. 12b) also correlated strongly with the PMBR. Previous work showing that passive movements produce a PMBR of equal amplitude to active movements, supports an afferent influence on the PMBR (Cassim et al., 2001). Our results cannot distinguish between the influence of motor efferents or somatosensory afferents on the PMBR.

\section{The effect of parameterisation}

The effect of parameterisation with the three movement conditions was to show a relation between PMBR strength and BOLD amplitude on an individual level for the motor BOLD response, with a strong correlation between motor BOLD amplitude and the PMBR strength (Fig. 7b), but not for the PMBR BOLD response (Fig. 12d). The differing effect of parameterisation in the two cases can be explained by considering the different sources of the BOLD activity and their relative contrast to noise ratios. The motor BOLD activity has a higher CNR than the PMBR BOLD activity, due to both the intrinsic higher contrast of the motor BOLD signal and the possible noisier coupling between oscillatory PMBR activity and the BOLD signal than that between motor activity and the BOLD signal.

\section{Location and function of the beta rebound}

Group analysis of all six subjects showed significant PMBR activation over both the motor cortex and somatosensory cortex, with the focus of activation located post-centrally. Analysis of individual data showed a post-central focus of PMBR activation for the three subjects with the strongest rebound. This is in contrast to the predominantly central or pre-central location found in a previous work using EEG and MEG (Salmelin et al., 1995; van Burik and Pfurtscheller, 1999). However, in one of these studies (van Burik and Pfurtscheller, 1999), the PMBR was located postcentrally in the somatosensory cortex for one out of only three subjects studied. Data from intracerebral recordings show a PMBR in both pre- and post-central regions, in closer agreement with our findings (Crone et al., 1998; Ohara et al., 2000; Szurhaj et al., 2003). One of these studies (Crone et al., 1998) also identified the strongest PMBR to be post-central.

The MEG study (Salmelin et al., 1995) identified both a $10 \mathrm{~Hz}$ (mu) and a $20 \mathrm{~Hz}$ rebound, with the $10 \mathrm{~Hz}$ rebound located as post- central but also strongly bilateral. It seems unlikely that we are sensitive to this mu rebound since, although post-central, there is no evidence for bilateral activity in our data. Furthermore, inspection of the time-frequency representations of the EEG data shows that only half of the subjects have a mu rebound, and this generally has a different time course to the beta rebound. In only one case (subject 3) is it possible that the beta rebound is a harmonic of the mu rebound. We do not therefore believe that the reported PMBR BOLD activity is a correlate of the mu rebound.

Our results may be biased towards central locations of the PMBR due to choice of $\mathrm{C} 3$ as the electrode from which to extract the GLM regressors for the fMRI analysis. Future work adopting a similar approach of using EEG signal as a regressor for fMRI analysis could consider using average signal from a number of electrodes, or multiple regressors from a number of electrodes.

Earlier studies locating the PMBR in the motor area (Salmelin et al., 1995; van Burik and Pfurtscheller, 1999) are compatible with the hypothesis that the PMBR reflects an active uncoupling of the motor network. Our results are not directly compatible with this, since we locate the PMBR post-centrally; a region which receives sensory input. On close inspection, the focus of our PMBR activation is in, or close to, the hand representation area in the somatosensory cortex (Maldjian et al., 1999; Francis et al., 2000), an area that is closely related to the motor hand area at a functional level. This supports the hypothesis that the PMBR reflects movement-related somatosensory processing (Cassim et al., 2001; Alegre et al., 2002). This activity in the somatosensory region could then still act to inhibit the motor cortex via corticocortical projections between the somatosensory and motor cortex, explaining the finding of reduced excitability of motor cortex neurons during this period (Chen et al., 1998).

\section{The physiological basis for a BOLD signal increase}

The increase in MRI signal with increased EEG beta power suggests that there is an increase in the mean rate of synaptic activity in the local neuronal population during the PMBR compared to resting baseline, and that this increase scales with beta power. This could be due to an increase in the mean rate of synaptic activity per unit volume, an increase in the amount of vasoactive substance released per synaptic event, or a combination of both. A number of recent modelling papers (Chawla et al., 1999, 2000; Jensen et al., 2005) suggest a link between increased synchrony and increased mean firing rate of local neuronal populations. If the region has reciprocal connections from the excitatory neurons, then an increase in mean firing rate will be coupled to an increase in mean synaptic activity (as is generally seen; Heeger et al., 2000), leading to a BOLD signal increase.

One paper looking specifically at beta oscillations (Jensen et al., 2005), suggests that it is the inhibitory interneurons which maintain the oscillations, with the sparse firing of excitatory cells timelocked to the rhythm of these inhibitory interneurons. The increased activity of the inhibitory neurons could increase the BOLD signal, although evidence for a link between inhibitory activity and the BOLD signal is currently inconclusive (Waldvogel et al., 2000; Attwell and Iadecola, 2002).

In support of our findings, recent work investigating eventrelated changes in the gamma band (Foucher et al., 2003) also showed increased BOLD signal with increases in gamma power. Results at lower frequency bands show the opposite behaviour, with BOLD signal decreases following increased synchronisation 
in the delta band during sleep (Czisch et al., 2004), and the alpha band during rest (Goldman et al., 2002; Laufs et al., 2003a,b; Moosmann et al., 2003). Increased synchronisation in the beta band during rest showed both regions of increased and decreased BOLD, but predominantly increased BOLD (Laufs et al., 2003a,b). Our results therefore fit in with the general pattern of BOLD increases associated with increased synchronisation in higher frequency beta and gamma bands, but decreases at lower frequencies.

A further study showed an increased BOLD signal response to two tasks that evoked decreases in beta power (Singh et al., 2002). However, as the authors mention, the decrease in power they saw in the beta band may have been accompanied by increased power in a higher frequency band, which could be the cause of the BOLD signal increase. Furthermore, it does not necessarily follow that the processes involved in an active desynchronisation of activity are the same as those producing increased synchronisation. Note that, although the power of our PMBR may be overestimated due to beta suppression during our baseline, this suppression cannot be the cause of the PMBR BOLD signal change which is due to activity change after movement offset. Any BOLD change due to pre-offset beta suppression would not be expected to extend beyond the motor activation. In addition, we found no correlation $(P>0.4)$ between pre-offset suppression and PMBR BOLD amplitude.

\section{Simultaneous versus separate EEG and fMRI recordings}

In this work, we attempted to record event-related oscillations simultaneously with MRI, which has only previously been achieved with interleaved recordings (Foucher et al., 2003). Compared to recordings from outside the scanner, the noise inside the scanner is increased five-fold, with the PMBR only visible in 2 out of 6 subjects (Fig. 2). The extra noise is caused by the presence of the scanning and pulse artefacts, which have not been completely removed. However, for this particular study, involving averaging of data across many trials, the use of data recorded in separate sessions is acceptable. We believe that this general procedure of combining EEG and fMRI data from separate sessions could provide useful information in a number of eventrelated studies. If the effect under study is believed to be stable over time, there may be little or no benefit from a simultaneous measurement, which will compromise data quality.

\section{Conclusion}

In this work, we attempted to identify a BOLD correlate of beta synchronisation following movement offset (the PMBR). By including an extra PMBR regressor in our general linear model, we have identified a region of PMBR BOLD activity in the sensorimotor cortex, with the most significant region close to the hand representation area in the post-central sulcus. In this region, the BOLD signal is extended in time compared to the region of motor activation, and the intersubject PMBR BOLD amplitude is shown to be positively correlated with PMBR strength. In addition, we found that BOLD activity during movement is strongly correlated with the PMBR EEG activity within individual subjects, suggesting an influence of motor cortex activity, or afferent somatosensory input on the subsequent PMBR.

More generally, our results show that event-related changes in oscillatory activity have an associated BOLD signal change. This work opens the way for similar combined EEG and fMRI studies of other event-related oscillatory behaviour.

\section{Acknowledgments}

We would like to thank Pascal Fries for the use of his multitaper software, Ole Jensen and Miranda van Turennout for helpful discussion and Erik van den Boogert and Ingmar Gutberlet for technical advice.

\section{References}

Alegre, M., Labarga, A., Gurtubay, I.G., Iriarte, J., Malanda, A., Artieda, J., 2002. Beta electroencephalograph changes during passive movements: sensory afferences contribute to beta event-related desynchronization in humans. Neurosci. Let. 331, 29-32.

Allen, P.J., Polizzi, G., Krakow, K., Fish, D.R., Lemieux, L., 1998. Identification of EEG events in the MR scanner: the problem of pulse artifact and a method for its subtraction. NeuroImage 8 (3), 229-239.

Allen, P.J., Josephs, O., Turner, R., 2000. A method for removing imaging artifact from continuous EEG recorded during functional MRI. NeuroImage 12 (2), 230-239.

Attwell, D., Iadecola, C., 2002. The neural basis of functional brain imaging signals. Trends Neurosci. 25 (12), 621-625.

Cassim, F., Monaca, C., Szurhaj, W., Bourriez, J.L., Defebvre, L., Derambure, P., Guieu, J.D., 2001. Does post-movement beta synchronization reflect an idling motor cortex? NeuroReport 12 (17), $3859-3863$.

Chawla, D., Lumer, E.D., Friston, K.J., 1999. The relationship between synchronization among neuronal populations and their mean activity levels. Neural Comput. 11 (6), 1389-1411.

Chawla, D., Lumer, E.D., Friston, K.J., 2000. Relating macroscopic measures of brain activity to fast, dynamic neuronal interactions. Neural Comput. 12 (12), 2805-2821.

Chen, R., Yaseen, Z., Cohen, L.G., Hallett, M., 1998. Time course of corticospinal excitability in reaction time and self-paced movements. Ann. Neurol. 44 (3), 317-325.

Crone, N.E., Miglioretti, D.L., Gordon, B., Sieracki, J.M., Wilson, M.T., Uematsu, S., Lesser, R.P., 1998. Functional mapping of human sensorimotor cortex with electrocorticographic spectral analysis: I. Alpha and beta event-related desynchronization. Brain 121, 2271-2299.

Czisch, M., Wehrle, R., Kaufmann, C., Wetter, T.C., Holsboer, F., Pollmacher, T., Auer, D.P., 2004. Functional MRI during sleep: BOLD signal decreases and their electrophysiological correlates. Eur. J. Neurosci. 20 (2), 566-574

Foucher, J.R., Otzenberger, H., Gounot, D., 2003. The BOLD response and the gamma oscillations respond differently than evoked potentials: an interleaved EEG-fMRI study. BMC Neurosci. 4, 22.

Francis, S.T., Kelly, E.F., Bowtell, R., Dunseath, W.J.R., Folger, S.E., McGlone, F., 2000. FMRI of the responses to vibratory stimulation of digit tips. NeuroImage 11 (3), 188-202.

Glover, G.H., 1999. Deconvolution of impulse response in event-related BOLD fMRI. NeuroImage 9 (4), 416-429.

Goldman, R.I., Stern, J.M., Engel, J., Cohen, M.S., 2002. Simultaneous EEG and fMRI of the alpha rhythm. NeuroReport 13 (18), $2487-2492$.

Heeger, D.J., Huk, C.H., Geisler, W.S., Albrecht, D.G., 2000. Spikes versus BOLD: what does neuroimaging tell us about neuronal activity? Nat. Neurosci. 3 (7), 631-633.

Jensen, O., Goel, P., Kopell, N., Pohja, M., Hari, R., Ennentrout, B., 2005. On the human sensorimotor-cortex beta rhythm: sources and modeling. NeuroImage 26 (2), 347-355. 
Laufs, H., Kleinschmidt, A., Beyerle, A., Eger, E., Salek-Haddadi, A., Preibisch, C., Krakow, K., 2003a. EEG-correlated fMRI of human alpha activity. NeuroImage 19 (4), 1463-1476.

Laufs, H., Krakow, K., Sterzer, P., Eger, E., Beyerle, A., SalekHaddadi, A., Kleinschmidt, A., 2003b. Electroencephalographic signatures of attentional and cognitive default modes in spontaneous brain activity fluctuations at rest. Proc. Natl. Acad. Sci. 100 (19), 11053-11058.

Logothetis, N.K., Pauls, J., Augath, M., Trinath, T., Oeltermann, A., 2001. Neurophysiological investigation of the basis of the fMRI signal. Nature 412 (6843), 150-157.

Maldjian, J.A., Gottschalk, A., Patel, R.S., Detre, J.A., Alsop, D.C., 1999. The sensory somatotopic map of the human hand demonstrated at 4 Tesla. NeuroImage 10 (1), 55-62.

Mathiesen, C., Caesar, K., Akgoren, N., Lauritzen, M., 1998. Modification of activity-dependent increases of cerebral blood flow by excitatory synaptic activity and spikes in rat cerebellar cortex. J. Physiol. 512 (2), $555-566$.

Mitra, P.P., Pesaran, B., 1999. Analysis of dynamic brain imaging data. Biophys. J. 76 (2), 691-708.

Moosmann, M., Ritter, P., Krastel, I., Brink, A., Thees, S., Blankenburg, F., Taskin, B., Obrig, H., Villringer, A., 2003. Correlates of alpha rhythm in functional magnetic resonance imaging and near infrared spectroscopy. NeuroImage 20 (1), 145-158.

Mugler, J.P., Brookeman, J.R., 1990. 3-Dimensional magnetization-prepared rapid gradient-echo imaging (3DMP-RAGE). Magn. Reson. Med. 15 (1), 152-157.

Neuper, C., Pfurtscheller, G., 2001. Event-related dynamics of cortical rhythms: frequency-specific features and functional correlates. Int. J. Psychophysiol. 43 (1), 41-58.

Ohara, S., Ikeda, A., Kunieda, T., Yazawa, S., Baba, K., Nagamine, T., Taki, W., Hashimoto, N., Mihara, T., Shibasaki, H., 2000. Movement-related change of electrocorticographic activity in human supplementary motor area proper. Brain 123, 1203-1215.

Pfurtscheller, G., Lopes da Silva, F.H.L., 1999. Event-related EEG/MEG synchronization and desynchronization: basic principles. Clin. Neurophysiol. 110 (11), 1842-1857.
Pfurtscheller, G., Stancak, A., Neuper, C., 1996. Post-movement beta synchronization. A correlate of an idling motor area? Electroencephalogr. Clin. Neurophysiol. 98 (4), 281-293.

Pfurtscheller, G., Zalaudek, K., Neuper, C., 1998. Event-related beta synchronization after wrist, finger and thumb movement. Electroencephalogr. Clin. Neurophysiol., Electromyogr. Motor Control 109 (2), $154-160$.

Rajapakse, J.C., Kruggel, F., Maisog, J.M., von Cramon, D.Y., 1998. Modeling hemodynamic response for analysis of functional MRI timeseries. Hum. Brain Mapp. 6 (4), 283-300.

Salmelin, R., Hamalainen, M., Kajola, M., Hari, R., 1995. Functional segregation of movement-related rhythmic activity in the human brain. NeuroImage 2 (4), 237-243.

Singh, K.D., Barnes, G.R., Hillebrand, A., Forde, E.M.E., Williams, A.L., 2002. Task-related changes in cortical synchronization are spatially coincident with the hemodynamic response. NeuroImage 16 (1) $103-114$.

Soltysik, D.A., Peck, K.K., White, K.D., Crosson, B., Briggs, R.W., 2004 Comparison of hemodynamic response nonlinearity across primary cortical areas. NeuroImage 22 (3), 1117-1127.

Stancak, A., Riml, A., Pfurtscheller, G., 1997. The effects of external load on movement-related changes of the sensorimotor EEG rhythms. Electroencephalogr. Clin. Neurophysiol. 102 (6), 495-504.

Szurhaj, W., Derambure, P., Labyt, E., Cassim, F., Bourriez, J.L., Isnard, J., Guieu, J.D., Mauguiere, F., 2003. Basic mechanisms of central rhythms reactivity to preparation and execution of a voluntary movement: a stereoelectroencephalographic study. Clin. Neurophysiol. 114 (1), $107-119$.

van Burik, M., Pfurtscheller, G., 1999. Functional imaging of postmovement beta event-related synchronization. J. Clin. Neurophysiol. 16 (4), $383-390$.

Waldvogel, D., van Gelderen, P., Muellbacher, W., Ziemann, U., Immisch, I., Hallett, M., 2000. The relative metabolic demand of inhibition and excitation. Nature 406 (6799), 995-998.

Yousry, T.A., Schmid, U.D., Alkadhi, H., Schmidt, D., Peraud, A., Buettner, A., Winkler, P., 1997. Localization of the motor hand area to a knob on the precentral gyrus-A new landmark. Brain 120, 141-157. 